\title{
POISSON BRACKETS AND COMMUTATOR BRACKETS. II
}

REESE T. PROSSER

\begin{abstract}
A previous result, that for certain problems in Hamiltonian mechanics the commutator bracket is determined by the Poisson bracket, is here extended to include certain problems defined in terms of current algebras.
\end{abstract}

A fundamental concept in the Hamiltonian theory of mechanics, whether classical or quantum, is that of the Poisson bracket, which determines the development of the system in time. In previous papers [1], [2] we have shown that a suitably defined form of Poisson bracket essentially determines the form of the commutator bracket, and hence the commutation relations, in the "moment algebra" generated by the canonical coordinates of the system. Here we shall show that this rather surprising result is in fact quite general and holds in any algebra without too many zero divisors. In particular, it holds in the moment algebra generated by the currents of a classical or quantum (Boson or Fermion) field, and hence offers the possibility of a unified approach to the Hamiltonian formulation of field theory.

Let $A$ be any (associative) algebra over the scalar field $F$. By a Poisson bracket for $A$ we mean a skew-symmetric bilinear derivation of $A$, i.e., a mapping $\{\}:, A \times A \rightarrow A$ satisfying

$$
\begin{aligned}
\{x, y\} & =-\{y, x\}, \\
\{x, \alpha y+\beta z\} & =\alpha\{x, y\}+\beta\{x, z\}, \\
\{x, y z\} & =\{x, y\} z+y\{x, z\} .
\end{aligned}
$$

One example of a Poisson bracket for $A$ is the usual commutator bracket [, ], given by

$$
[x, y]=x y-y x
$$

It follows from our principal result here that for a large class of algebras this is essentially the only example.

Received by the editors March 30, 1976.

AMS (MOS) subject classifications (1970). Primary 81A20. 
To formulate our result we need just one definition: If $E$ is any subset of $A$ we denote by $E^{\perp}$ the annihilator of $E$ :

$$
E^{\perp}=\{x: x y=y x=0 \text { for all } y \in E\} .
$$

If $A$ has a Poisson bracket $\{$,$\} then we denote by \{A, A\}$ the finite linear span of all elements of the form $\{x, y\}$, for $x, y \in A$.

Now we can state

THEOREM 1. Let $A$ be an associative algebra over the field $F$ and let $\{$,$\} be a$ Poisson bracket for A. Assume

$$
\{A, A\}^{\perp}=\{0\} .
$$

Then $A$ may be isomorphically embedded in a larger algebra $B$ (obtained by adjoining a single central indeterminate $z$ ) such that in $B$ the commutator bracket $[$,$] is given by$

$$
[x, y]=z\{x, y\}
$$

Condition (6) says that the range of the Poisson bracket does not annihilate anything in $A$. This condition is met, in particular, if the range contains some non-zero-divisor in $A$; if $A$ has no zero-divisors, then (6) says simply that the Poisson bracket is not identically zero.

The proof depends entirely on the following identity:

LEMMA 2. For all $x, y, z, w \in A$ we have

$$
\{x, y\}[z, w]=[x, y]\{z, w\} .
$$

Proof. Consider the expression $\{x z, y w\}$. We expand it in two ways and compare:

$$
\begin{aligned}
\{x z, y w\} & =\{x z, y\} w+y\{x z, w\} \\
& =\{x, y\} z w+x\{z, y\} w+y\{x, w\} z+y x\{z, w\} . \\
\{x z, y w\} & =\{x, y w\} z+x\{z, y w\} \\
& =\{x, y\} w z+y\{x, w\} z+x\{z, y\} w+x y\{z, w\} .
\end{aligned}
$$

Subtracting (10) from (9), we obtain

$$
0=\{x, y\}[z, w]-[x, y]\{z, w\},
$$

as required.

Now we recall that every bilinear mapping from $A \times A$ to $A$ can be considered as a linear mapping from the tensor product $A \otimes A$ to $A$. This is true, in particular, for both the Poisson and commutator brackets. The next lemma says that the kernel of the Poisson bracket in $A \otimes A$ is included in the kernel of the commutator bracket. 
LEMMA 3. For every finite set $x_{i}, y_{i} \in A$, we have that if $\sum_{i}\left\{x_{i}, y_{i}\right\}=0$ then $\Sigma_{i}\left[x_{i}, y_{i}\right]=0$.

Proof. Choose $u_{j}, v_{j} \in A$ and consider

$$
0=\left(\sum_{i}\left\{x_{i}, y_{i}\right\}\right)\left(\sum_{j}\left[u_{j}, v_{j}\right]\right)=\left(\sum_{i}\left[x_{i}, y_{i}\right]\right)\left(\sum_{j}\left\{u_{j}, v_{j}\right\}\right) .
$$

Here we have used (8) and the hypothesis. Now by (6) we conclude

$$
\sum_{j}\left[x_{i}, y_{i}\right]=0 \text {. }
$$

Now we know that because of Lemma 3 the (linear) mapping [, ]: $A \otimes A$ $\rightarrow A$ can be factored through $\{$,$\} :$

COROLlARY 4. Under the hypothesis of Theorem 1 there exists a linear mapping ': $\{A, A\} \rightarrow[A, A]$ such that, for all $x, y \in A$

$$
\{x, y\}^{\prime}=[x, y] .
$$

Next we extend the mapping ' from $\{A, A\}$ to the two-sided ideal $I$ in $A$ generated by $\{A, A\}$. To do this we need

Lemma 5. If $x \in A, y \in\{A, A\}$ and $x y \in\{A, A\}$, then

$$
x y^{\prime}=(x y)^{\prime}, \quad y^{\prime} x=(y x)^{\prime} .
$$

Proof. Choose $w \in\{A, A\}$, and note that by (8)

$$
y^{\prime} w=y w^{\prime}
$$

Hence

$$
x\left(y^{\prime} w\right)=x\left(y w^{\prime}\right)
$$

Similarly

$$
(x y)^{\prime} w=(x y) w^{\prime} .
$$

Now since $A$ is associative, we have

$$
\left((x y)^{\prime}-x y^{\prime}\right) w=0
$$

and by (16) we obtain (15), as required.

Because of (15) we can now extend the mapping' from $\{A, A\}$ to $I$ $=A\{A, A\} A$, by simply requiring that 'commute with left and right multiplication, i.e., by requiring (15) for any $x \in A, y \in\{A, A\}$.

To complete the proof of Theorem 1 we need only realize the mapping ' by multiplication by a suitable central element $z$. The immediate difficulty is that such a $z$ may not lie in $A$. But we can always adjoin it as follows: 
Let $A[z]$ be the algebra obtained from $A$ by adjoining a single central indeterminate $z$. Here it is understood that $z$ commutes with everything in $A$, and the elements of $A[z]$ may be taken as polynomials in $z$ with coefficients in $A$ subject only to the relations obtained from $A$. Note that $A$ is naturally contained in $A[z]$ as the constants.

We extend the Poisson bracket $\{$,$\} from A$ to $A[z]$ by requiring, for all $x \in A$,

$$
\{z, x\}=0
$$

It follows from (3) that

$$
\left\{z^{m} x, z^{n} y\right\}=z^{m+n}\{x, y\} .
$$

Because $z$ commutes with everything we also have

$$
[z, x]=0
$$

and hence

$$
\left[z^{m} x, z^{n} y\right]=z^{m+n}[x, y] .
$$

It follows that we can extend the mapping 'from $A\{A, A\} A$ to $A[z]\{A, A\} A[z]$ by the prescription

$$
\left(z^{m} u w v z^{n}\right)^{\prime}=z^{m} u w^{\prime} v z^{n}
$$

and by linearity. Then we can state

LeMMA 6. Let $J$ be the subset of $A[z]$ defined by

$$
J=\left\{x^{\prime}-z x: x \in A[z]\{A, A\} A[z]\right\} .
$$

Then $J$ is a two-sided ideal in $A[z]$, and $J \cap A=\{0\}$.

Proof. We need only show, for $x^{\prime}-z x$ and $y^{\prime}-z y$ in $J$, that

$$
\left(x^{\prime}-z x\right)+\left(y^{\prime}-z y\right)=x^{\prime}+y^{\prime}-z(x+y)
$$

lies in $J$, and for $u, v \in A$ that

$$
z^{m} u\left(x^{\prime}-z x\right) v z^{n}=z^{m} u x^{\prime} v z^{n}-z\left(z^{m} u x v z^{n}\right)
$$

lies in $J$. But the first of these follows from the linearity of the mapping ', and the second from (24). Since no nonzero element of $J$ is independent of $z, J \cap A=\{0\}$.

To complete the proof of Theorem 1, we now have only to define the algebra $B$ by

$$
B=A[z] / J .
$$

Then $B$ is an algebra, $A$ is embedded in $B$ isomorphically (since $A \cap J$ 
$=\{0\})$, and in $B, x^{\prime}=z x$ whenever $x^{\prime}$ is defined. In particular, $[x, y]=\{x, y\}^{\prime}$ $=z\{x, y\}$, as required.

Several special cases of Theorem 1 are of particular interest. If $A$ is finitely generated, with generators $x_{i}$ such that $\left\{x_{i}, x_{j}\right\}=\lambda_{i j} 1$, with $\lambda_{i j}$ not all zero, then $\lambda_{i j} 1$ lies in $\{A, A\}$, and (6) holds. It follows that for any $f, g \in A$ we have $[f, g]=z\{f, g\}$ where $z=\lambda_{i j}^{-1}\left[x_{i}, x_{j}\right]$ already lies in $A$. This is the case if $A$ is the moment algebra generated by the canonical coordinates of an $n$-particle mechanical system; for a classical system $z=0$, and for a quantum system $z=i \hbar 1$.

If, instead of $\left\{x_{i}, x_{j}\right\}=1$, we require $\left\{x_{i}, x_{j}\right\}=\sum_{k} c_{i j}^{k} x_{k}$, in such a way that the generators form a semisimple Lie algebra under $\{$,$\} , then the x_{i}$ lie in $\{A, A\}$, and (6) holds providing that $A$ contains no elements $w \neq 0$ with $w^{2}=0$. It follows that for any $f, g \in A$ we have $[f, g]=z\{f, g\}$ where $z$ may not lie in $A$. This is the case, for example, if $A$ is generated by the symmetric 2nd degree homogeneous polynomials $f_{i j}=x_{i} x_{j}+x_{j} x_{i}$ in the canonical coordinates of the $n$-particle system above. These polynomials form the Lie algebra of the symplectic group under $\{$,$\} if the matrix \lambda_{i j}$ is nondegenerate. In this case the element $z=\lambda_{i j}^{-1}\left[x_{i}, x_{j}\right]$ does not lie in $A$ but must be adjoined. The resulting algebra $B$ is that generated by all 2 nd degree homogeneous polynomials $g_{i j}=x_{i} x_{j}$.

A similar situation obtains if $A$ is generated by the skew-symmetric 2nd degree homogeneous polynomials $f_{i j}=x_{i} x_{j}-x_{j} x_{i}$ in the canonical coordinates of a system subject to the anticommutation relations $x_{i} x_{j}+x_{j} x_{i}=\lambda_{i j} z$. These polynomials $f_{i j}$ then form the Lie algebra of the orthogonal group under $\{$,$\} if the matrix \lambda_{i j}$ is nondegenerate. Again in this case the element $z$ must be adjoined, and the resulting algebra is that generated by all 2 nd degree homogeneous polynomials.

These examples suggest the possibility of a reformulation of quantum field theory in terms of currents. If $\varphi(\underline{x})$ denotes a (Boson or Fermion) quantum field and $h_{i}(x)$ is a complete orthonormal basis for $\mathfrak{L}^{2}(\underline{x})$, then the field elements $\varphi_{i}=\int \varphi(x) h_{i}(x) d x$ will generate a moment algebra [1]. The subalgebra generated by the currents $\varphi_{i} \varphi_{j}$ should admit a Poisson bracket which determines the development of the system in time. To specify this bracket on the currents is, according to our theorem, to specify the commutation relations among the currents, and hence among the fields, up to some central element. In any irreducible representation of the moment algebra this central element becomes a scalar and the choice of this scalar determines the dynamics of the system.

We close with a corollary.

COROllary 7. If, in Theorem 1, we assume

$$
[A, A]^{\perp}=\{0\}
$$


then $A$ may be embedded isomorphically in a larger algebra $C$ (obtained by adjoining a single central indeterminate w) such that in $C$ the Poisson bracket $\{$, is given by

$$
\{x, y\}=w[x, y]
$$

Proof. Interchange the role of $\{$,$\} and [$,$] throughout.$

Thus in algebras satisfying (27) the Poisson bracket is essentially unique.

\section{BIBLIOGRAPHY}

1. R. T. Prosser, A new formulation of particle mechanics, Mem. Amer. Math. Soc. No. 61 (1967), 57 pp. MR 32 \#8545.

2. - Poisson brackets and commutator brackets. I, Proc. Amer. Math. Soc. (submitted).

Department of Mathematics, Dartmouth College, Hanover, New Hampshire 03755 